\title{
Optimum Space Utilization Approach to Improve Persistence of WSNs
}

\author{
Anuja Modi \\ Computer Science and Engineering \\ VNS Institute of Technology \\ Bhopal,India \\ modi.anuja15@gmail.com \\ Gourishankar Prajapati \\ Head of Department Computer Science and Engineering \\ VNS Faculty of Engineering \\ Bhopal,India \\ gsprajapati1234@gmail.com
}

\begin{abstract}
Wireless Sensor Networks (WSNs) are consists of huge number of sensor nodes, restricted with limited power, for provide services with cooperation to sense data and send to server. Various routing techniques are introduced for managing data transmission in WSNs in last decade. This work proposes novel method with compare distance of sensor nodes to base station in heterogeneous WSN. The sensor nodes near to base station are likely to send data direct to base station and nodes are long distance to base station would form cluster to send data using Stable Election Protocol (SEP) method. This work considers distance parameter to compare with base station position to decide whether need for nodes clustering or not. The proposed method further compare with existing Low Energy adaptive clustering hierarchy (LEACH) protocol and SEP protocol for WSN data transmission. The proposed method simulation results show the effectiveness with compare to existing methods.
\end{abstract}

\section{Index Terms}

Distance based Stable, Election, Protocol, Wireless, Sensor, Networks.

\section{Council for Innovative Research}

Peer Review Research Publishing System

Journal: INTERNATIONAL JOURNAL OF COMPUTERS \& TECHNOLOGY

Vol. 15, No. 1

www.ijctonline.com, editorijctonline@gmail.com 


\section{INTRODUCTION}

WSNs are network designed by deploy various sensor elements spread random manner without predefine location for collecting different type of data as need for different applications. Different type of data may be collected as pressure, motion heat, noise, vibrations, or contaminant from special environmental site somewhere human being unable to go for read and collecting data. As well as technology developed in area of wireless communication this technology used for improve WSN also. The latest WSN are low cost and more efficient which help various fields such as health monitoring system for patients to collect biological data, home security to sense fire or other unwanted sensing data, etc. Large numbers of study had done in this field of WSNs for defeat various technical problems within given application on various stages. Sensor nodes consists of hardware and software's those are able sense information data, dealing with capture data and also can communicate with other nodes to further send or receive data. The system of such type of WSN need to self organized according to conditions for reliable delivery of data to base station with effective functionality.

WSN uses different method for transmit information this method varies according to situations of network requirements. Normally there are three basic methods used-

- Clock Driven: Nodes in network collect information required and send according to predefine time interval.

- $\quad$ Event Driven: In this case data from sensor node to base station whenever special even happened.

- Query Driven: Data transmission take place when ever any query generated regarding data capturing

In mention all types of data transmission, resourceful utilization of energy is worry during learning, planning or installations of this type networks to extend the sensing time and the whole life span of the network.

Hierarchical routing methods have been establishing additional energy proficient routing methods. Numerous protocols are deliberate for standardized networks. LEACH [1] is original grouped supported routing algorithm applied on homogeneous type network. LEACH allocates equivalent possibility for every node to be performed as cluster head of current group. Still, LEACH does not achieve best performance in heterogeneous situation. Heterogeneity of nodes through value to their battery energy point has as well showed additional lifetime for WSNs. For enhance energy effectiveness of WSNs, SEP [2] was given on WSNs. SEP has more levels such as two levels for maintain energy of nodes in heterogeneous situations. SEP allocates different chance to be any node cluster head on the according to their energy level. Yet, SEP not implies additional energy of upper level nodes proficiently.

For sending information to base it perform within minimum use of energy. To do this there is need to good routing protocol algorithm. Such protocol must help to proper energy utilization for each node in WSN. Traditional methods are not capable to complete this task. So here is need of hybrid approach which can perform efficient energy utilization by combining more than one method together for improvement of stability period, network lifespan and also efficiency of the network.

\section{RELATED WORK}

WSN need to be scale as required in such conditions hierarchical clustering is better way to deal with data transmission. Hierarchical techniques have extra benefit as efficient communication with good energy utilization. Such type of applications nodes having extra energy than normal nodes used to send sensed information. There are various protocol have been given such as LEACH [3], TEEN [5], APTEEN [6], PEGASIS[4] these method used advance node for better energy consumption.

Data load transmitted over network is very essential parameter for any network. This parameter is check and optimized during work on paper [7] by authors as clustering with load balancing for WSN network. Author verify density of cluster according to distance from base station. Hence if nodes are near to base station than density of clusters are low and for nodes are long distance from base station are from cluster with more density to optimum energy use. This method improved total life time of network.

Another previous technique consider more than one parameters as WCA [8] single hop distance. Cluster head selection is base on different parameters like one hop neighbors, remaining battery power and speed to node movement. This method need to calculate weight factor during each round hence need more power for calculation. Hence extra effort has to apply for cluster formation this overhead control the efficient of WCA method.

Basic method used for clustering sensor nodes k-clustering [9] used most k-hop neighbors to form cluster.

The ESAC [10] method uses main advantages of above mention methods and make more effective clustering method. This method uses cluster size as requirement of WSN condition. This restricts to create large size cluster to efficient clustering up-to two hop node distances. If cluster head died than new cluster head selected from this cluster member only. So new cluster formation take place only within current members other nodes are not affected by this cluster head selection. But two-hop distance clustering is not good idea for cluster formation.

Another author in paper [11] gives new technique to load balance within a cluster. This method maintains cluster density distribution according to distance from base station it basically completely different from the previous clump algorithms. This method better utilize according to distance but sue to lack of advance normal node are died very early stage.

LEACH [1] could be a ranked cluster algorithmic rule for considered usage of energy within the network. LEACH uses irregular rotation of the nearer cluster head. LEACH performs well in uniform conditions. In LEACH each node has same chance to become a cluster head. However, LEACH isn't compatible for heterogeneous conditions. SEP could be a two 
level heterogeneous protocol introducing two kinds of nodes, traditional nodes and advance nodes. Advance nodes have a lot of energy than traditional nodes. In SEP each nodes ([2] advance nodes) have weighted chance to become cluster head. Advance nodes have a lot of probabilities to become cluster head than traditional nodes. SEP doesn't guarantee economical installation of nodes.

\section{TERMINOLOGIES USED}

Few terms are used in this work these are explain here

- Stability Period: this is just Time interval of time at which network start and the time at which first sensor node dead.

- Constancy Period: difference of Time between death of first sensor node and time at last sensor node dead.

- Throughput: Throughput is just total data rate of at which information sent within network, it is rate of data transmitted from cluster heads till base station along with rate of data transmitted to base from normal sensor nodes.

- Network Lifespan: Time difference between time start of the network and time at which death of the last alive node.

- Phase: integer amount of cycles after which a node are capable to choose as cluster head.

- Data Collection: nodes within a nearer distance generally share almost same data to base station. So there should be restriction in such situations for reduce energy expenditure is data aggregation. Collection having contains redundancy in unusual information communication. While the redundancy is accomplished by a few signal processing systems, this procedure is known as data fusion.

\section{Proposed Sink Distance Based Protocol}

In SEP [2] traditional nodes and advance nodes area unit deployed arbitrarily. If majority of traditional nodes area unit deployed isolated from base station it consumes a lot of energy whereas sending information which ends up within the shortening of stability interval and reduce in efficiency. Therefore throughput of SEP decreases. To get rid of these flaws need to divide network field in regions. As corners may be most distant areas within the field, wherever nodes required extra energy to transmit information till base station. thus traditional nodes may placed close to the base station and they transmit their information directly to base station. but advance nodes may deployed long far away from base station as they thus extra energy. If advance nodes transmit information directly to base station extra energy consumes, hence for save energy of advance nodes cluster technique is implemented on advance nodes alone.

The proposed method is advancement of SEP scheme. This proposed work pursues hybrid mechanism i.e. direct data communication and data communication using cluster head. Here detail discussion is given with the functioning of proposed work.

\section{A. Network Architecture:}

Most of the routing protocols nodes are place in random manner within the network area thus overall energy of nodes can't be utilized properly. This work modified such existing method and considers network area as two regions: region 0 , Head region 1 , by energy levels of nodes and $X$ co-ordinate of network field. This is assumed that some ratio of node from all nodes having more energy level to compare with others. Suppose $m$ is ratio of total nodes $n$, having a time more energy power than other nodes. Let these nodes called advance nodes, hence $(1-m) \times n$ is quantity of normal nodes.

Region 0: Normal nodes are placed in random manner in Region 0 near area of base station, lying between $30<X<80$.

Head region 1: Half of advance nodes are deployed randomly in this region, lying between $0<X<=20$ and $80<X<=100$.

This placement is important because advance nodes having more energy levels than normal nodes and sides are most far away distance from center position of base station so if any nodes wants to send information to base station it have to spend more energy. Hence more energy level nodes placed such area Head Region 1. 


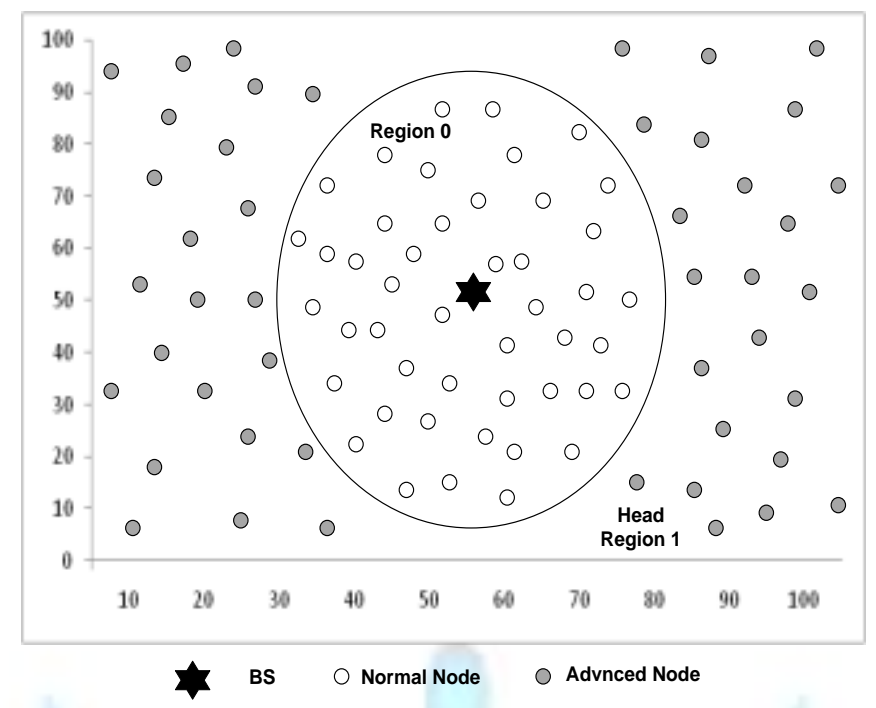

Fig. 1. Network Architecture

\section{B. Distance Based Protocol function}

Distance based protocol employ two methods to send information to base station. methods are:

- Direct transmission.

- Communication using Cluster head.

a) Direct Transmission

Nodes in Region 0 transmit information directly to base station. Normal nodes sense atmosphere, collected information of concern and transmit this data directly to base station.

b) Transmission via Cluster head

Nodes in Head region 1 send information to base station by applying clustering technique. Cluster head is chosen between nodes in Head region 1. Cluster head gather information from member nodes, comprehensive it and then sends it to base station. Cluster head election is main task and important function. Fig. 1 give all detail regarding WSN network node placement here advanced node are represented in dark color and normal node are shown in white color. Advance nodes are placed in head region 1 to utilize their battery power properly and normal nodes are placed nearby base station to save their battery power for increase network life span. Always Cluster is created only for advance nodes communication for base station.

Here consider that most favorable number of clusters Kopt and $\mathrm{n}$ is the number of advance nodes to minimize over heads. As mention in SEP optimal probability for cluster formation to decide cluster head by following formula using Kopt and $\mathrm{n}$ can given as

$$
P_{o p t}=\frac{K_{o p t}}{n}
$$

Each node chooses whether to be converted into cluster head within ongoing phase or not. Any random number among 0 and 1 is produced for node de. If generated random no. is a smaller than or equivalent to threshold $T(n)$ for a node then this node is selected as cluster head of current cluster. Threshold $T(n)$ can be express by following equation

$$
T(n)=\left\{\begin{array}{l}
\frac{P_{\text {opt }}}{1-P_{\text {opt }}\left(r \times \bmod \frac{1}{P_{\text {opt }}}\right)} \\
0 \quad \text { otherwise }
\end{array} \quad \text { if } n \in G\right.
$$

Where $G$ is the group of all nodes could not be cluster heads during last 1/Popt phases. Probability that any advance node to selected as cluster head as given in [2] can be express as

$$
P_{a d v}=\frac{P_{o p t}}{1+(\alpha . m)} \times(1+\alpha)
$$

By above equation threshold value for advance nodes can be given as

$$
T(n)=\left\{\begin{array}{l}
\frac{P_{a d v}}{1-P_{a d v}\left(r \times \bmod \frac{1}{P_{a d v}}\right)} \\
0 \quad \text { otherwise }
\end{array} \quad \text { if } a d v \in G^{\prime}\right.
$$


G' is the group of all advance nodes could not be cluster heads during last 1/Popt phases.

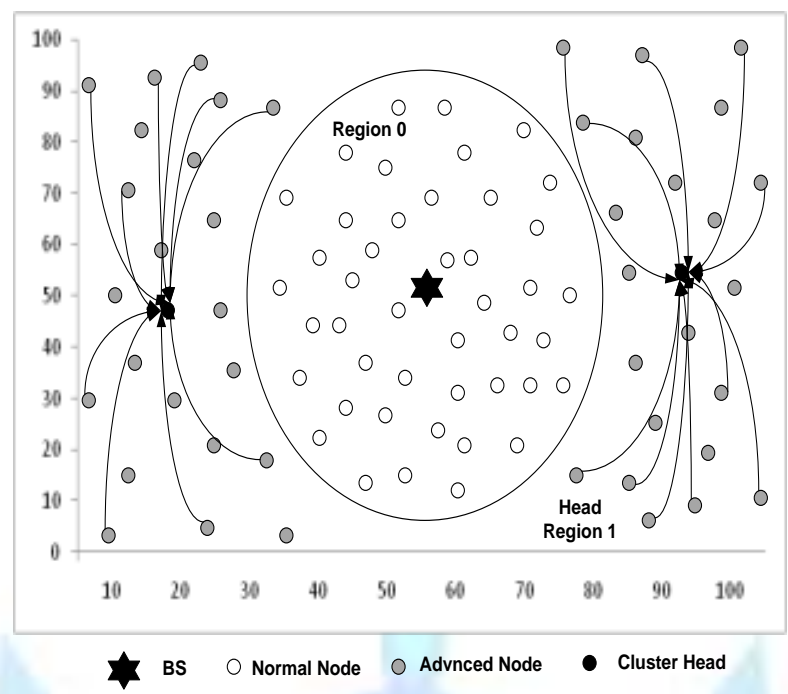

Fig. 2. Nodes sending data to cluster head

When cluster head is identified then it broadcasts a message to inform all nodes. All nodes within range of cluster head received information message and decide to which cluster node is head for current phase. This process is known as cluster formation phase. Nodes respond to cluster head for which get maximum signal strength and connect as cluster member of cluster head. Cluster head allocate TDMA slot as per scheduling algorithm for member nodes with help to these slots member send its data to cluster head. As cluster formation process is finished each member node gather information and transmitted it to cluster head within time slot allocated by cluster head. This detail process is given in fig. 2.

After receiving sensed information from all member nodes, Cluster head combined this data and then transmit this data to the base station this process is called as transmission phase. Fig. 3 demonstrates this process.

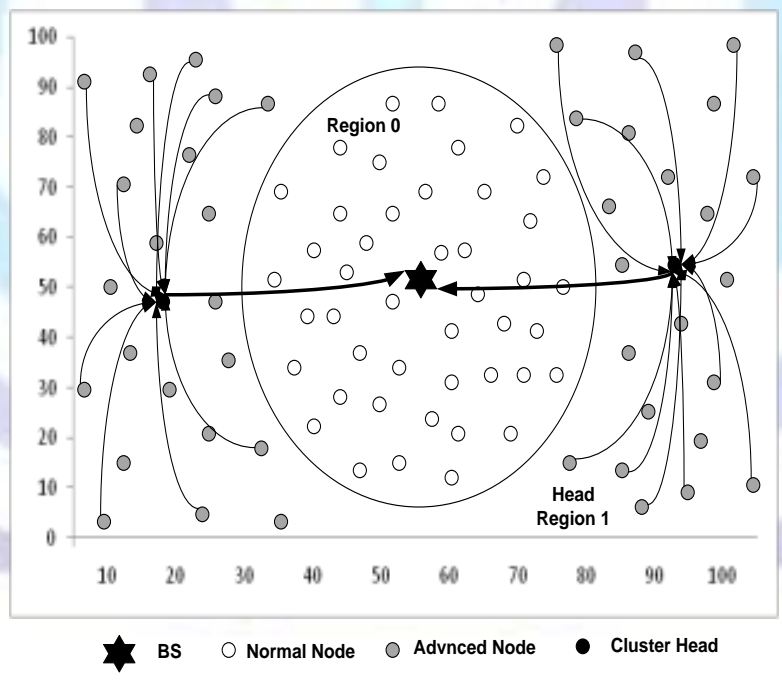

Fig. 3. Cluster head transmitting data to base station

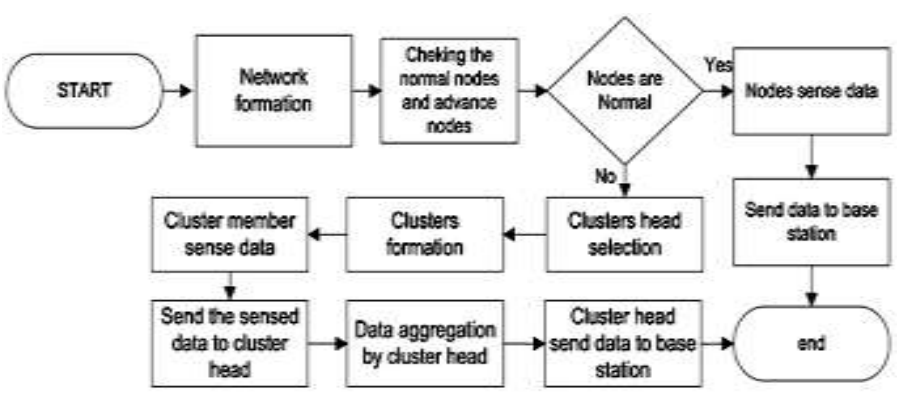

Fig. 4. Flow chart of Distance Based procedure 
The normal nodes (placed in Region 0) are no need to form cluster because they have lesser energy compare to advance nodes energy and if any normal node become cluster head then it require more energy to schedule and transmit all members' data to base station. If any normal nodes become cluster head it will dead very soon and this leads to shortening of consistency period. Overall network lifetime will be very short. Fig.4 consist proposed work operation in flowchart form.

\section{SIMULATIONS}

This proposed work performed within a network area having dimensions 100m height and 100m width, 100 nodes are placed in predefine regions according to their energy level. It is assumed that the Base station is situated at center of the network area. This work employs the first order radio model same as mentioned in traditional SEP scheme. For result analysis and graph comparison MATLAB is used to implement proposed work as simulator. Some predefine settings are considered for WSNs network as following. Here $20 \%$ of nodes are form as advance nodes and they are placed in Head region 0 . While Popt is set to 0.1 hence there should be 2 cluster heads per round. One cluster head selected from left side area and other cluster head is selected from right side area during each phase. All Other necessary parameters are given in Table 1.

TABLE I. SIMULATION PARAMETERS

\begin{tabular}{|l|l|}
\hline Parameters & Value \\
\hline Initial energy Eo & $0.5 \mathrm{~J}$ \\
\hline Initial energy of advance nodes & Eo( $(1+\alpha)$ \\
\hline Energy for data aggre gation EDA & $5 \mathrm{~nJ} / \mathrm{bit} /$ signal \\
\hline Transmitting and receiving energy Eelec & $5 \mathrm{~nJ} / \mathrm{bit}$ \\
\hline Amplification energy for short distance Efs & $10 \mathrm{Pj} / \mathrm{bit} / \mathrm{m} 2$ \\
\hline Amplification energy for long distance Eamp & $0.013 \mathrm{pJ} / \mathrm{bit} / \mathrm{m} 4$ \\
\hline Probability Popt & 0.1 \\
\hline
\end{tabular}

\section{Result ANd Discussion}

This segment discussed about result generated by proposed method during simulation on Matlab tool for WSN network and results are comparing with SEP and LEACH methods result generated on same platform. These results are implemented within heterogeneous environment and all parameters kept same for all proposed, SEP and LEACH method to get true results. The main objective of this simulation is to check total lifetime span and consistency of proposed method with comparison to others.

Figure 5 and Figure 6 demonstrate the results generated during network analysis with $m=0.2$ and $a=1$. Here $m$ stands for ration of advance node with total number of nodes within network. And a means percentage of extra energy within advance node in compare to normal node. According to this simulation network have 20 advance nodes out of total 100 nodes in network and remaining 80 nodes are with normal energy level this normal energy nodes are not participating in clustering method.

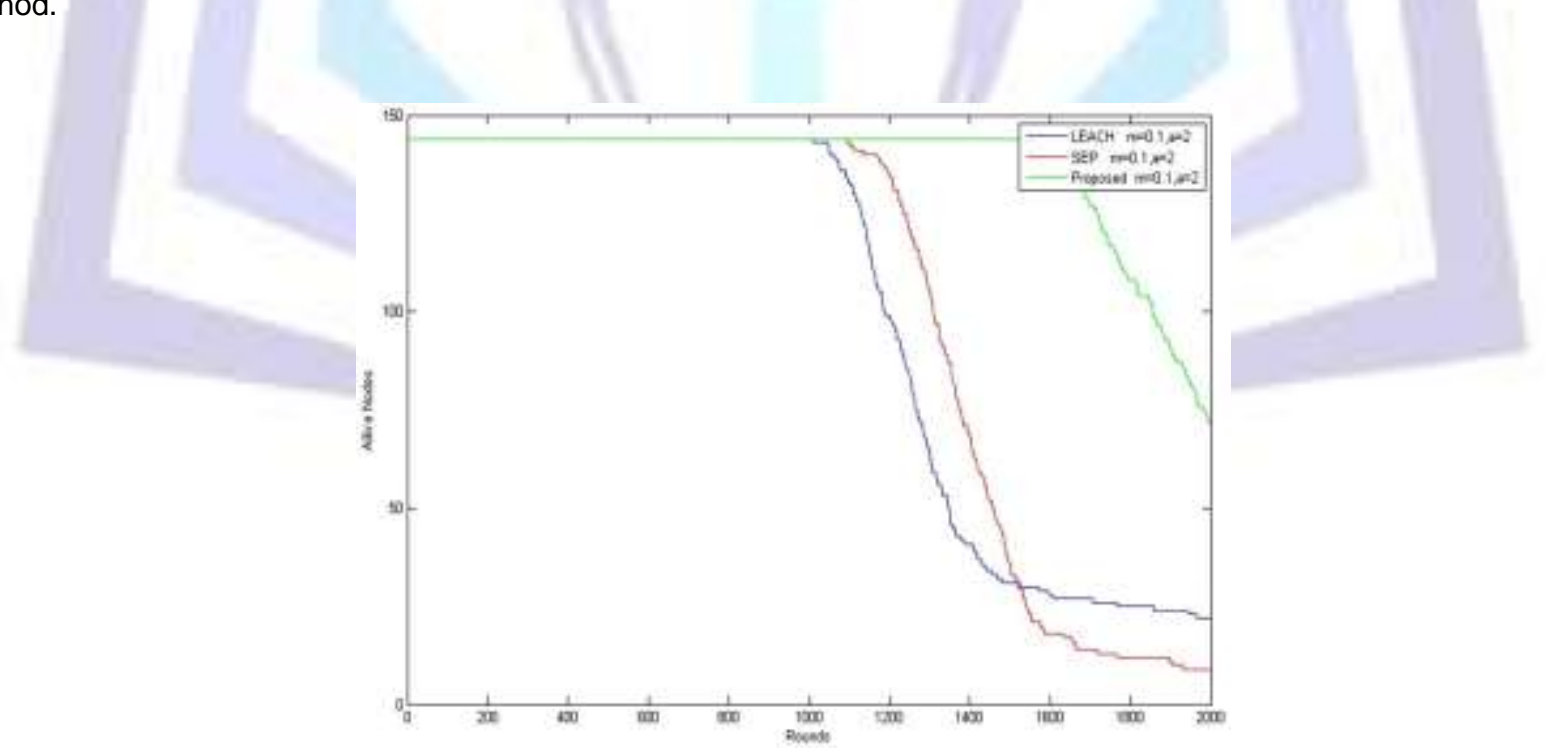

Fig. 5. Alive nodes in LEACH, SEP and proposed method

Figure 5 shows overall alive nodes within network after all maximum rounds completion. As graph shows the proposed method generates better result in compare to others. After completion of 1500 rounds first node dead during proposed method.

The well known about LEACH protocol is that it is very poor within heterogeneous conditions hence sensor nodes are exhaust their total energy and dead at very early stages and node dead with very fast rate. But SEP protocol performs quite good in heterogeneous network. Apart this SEP protocol does not select effectively. This method selects $\mathrm{CH}$ whether its 
normal or advance node with same probability hence SEP is not sufficient to maintain consistency of network. As result all normal nodes dead in starting stage and overall network has suffered.

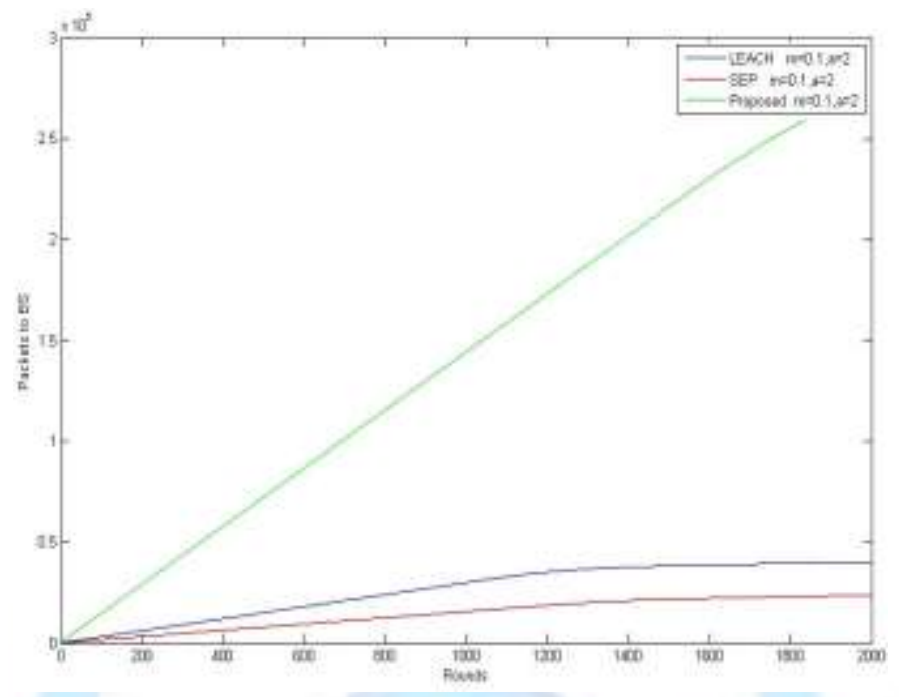

Fig. 6. Throughput of LEACH, SEP and proposed scheme

Figure 6 explains the total number of packets transmits to base station by all sensor nodes during proposed method. The proposed method outperforms well in compare to LEACH and SEP because of normal node used to directly send data base station.

TABLE II. COMPARISON TABLE WHEN M=0.2 AND A=1

\begin{tabular}{|l|l|l|l|}
\hline Protocol & $\begin{array}{l}\text { Stability } \\
\text { Period } \\
\text { (Rounds) }\end{array}$ & $\begin{array}{l}\text { Network } \\
\text { Lifetime } \\
\text { (Rounds) }\end{array}$ & $\begin{array}{l}\text { Throughput } \\
\text { (Packets) }\end{array}$ \\
\hline LEACH & 1018 & 4685 & $1.99 \times 104$ \\
\hline SEP & 1089 & 3005 & $3.43 \times 104$ \\
\hline proposed method & 1531 & 4119 & $2.21 \times 105$ \\
\hline
\end{tabular}

Figure 7 and Figure 8 demonstrate results generated by proposed protocol on setting different parameters $\mathrm{m}=0.1$ and $\mathrm{a}=2$. During this experiment total number of advance nodes in network are 10 with extra energy value of $a=2$.

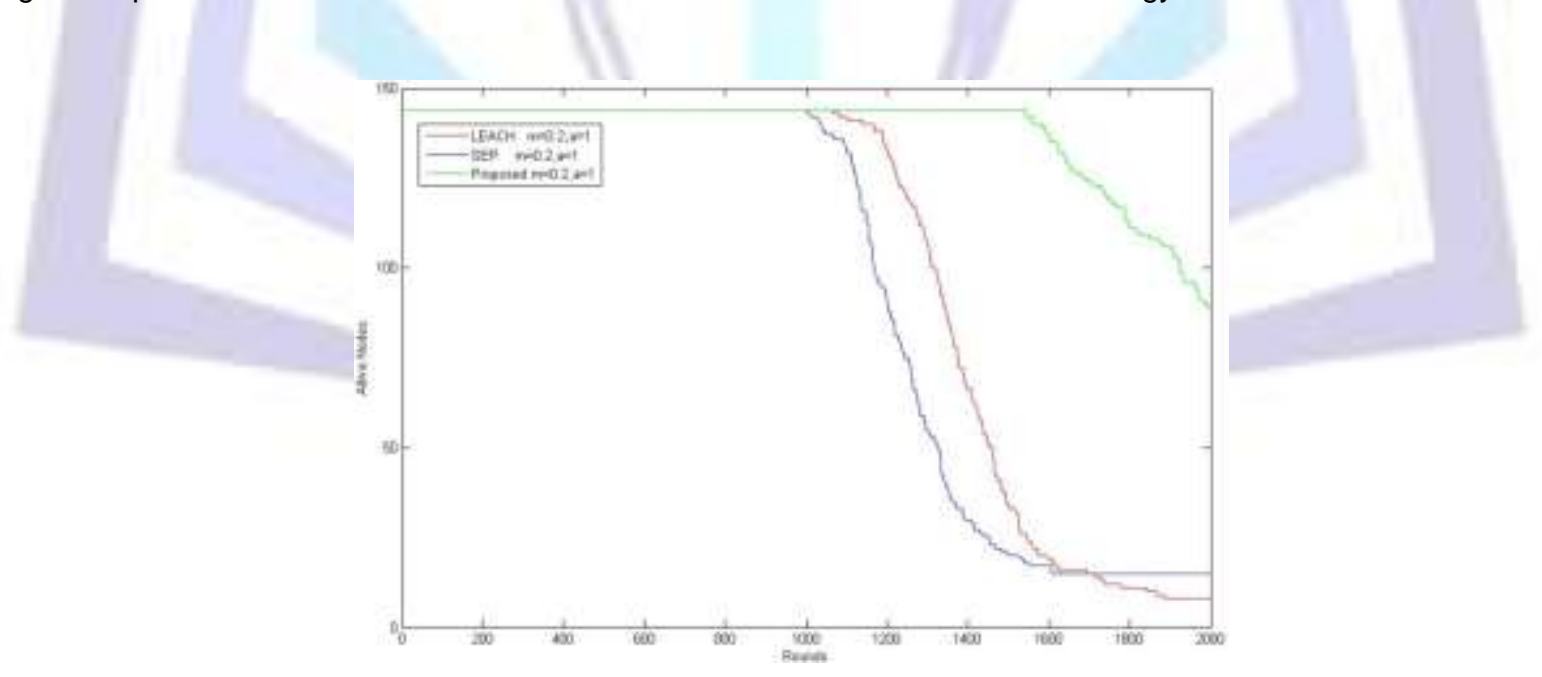

Fig. 7. Alive nodes in LEACH, SEP and proposed method

The Table 2 and Table 3 show the result comparisons of proposed method with other LEACH and SEP algorithms.

From Fig.7, we can see that stability time of proposed technique is roughly similar for both cases i.e. $(m=0.2, \alpha=1$ and $m=0.1, a=2$ ). The cause following is that normal nodes contain equal quantity of energy, they utilize equal quantity of energy and they expire about at the equal time as earlier, still network life span is better for the reason of the additional energy of advance nodes contains.

Constancy period of LEACH is reduced the reason that LEACH is very responsive to heterogeneity. LEACH does not have weighted scheme as in SEP in favor of even placement of extra energy nodes. In LEACH each node has equivalent 
possibility of becoming cluster head thus normal nodes dead earlier than advance nodes. Fig. 8 demonstrates throughput for LEACH, SEP and Distance Based method. Throughput of proposed method is better in compare to LEACH and SEP even though energy of advance node has been enlarged.

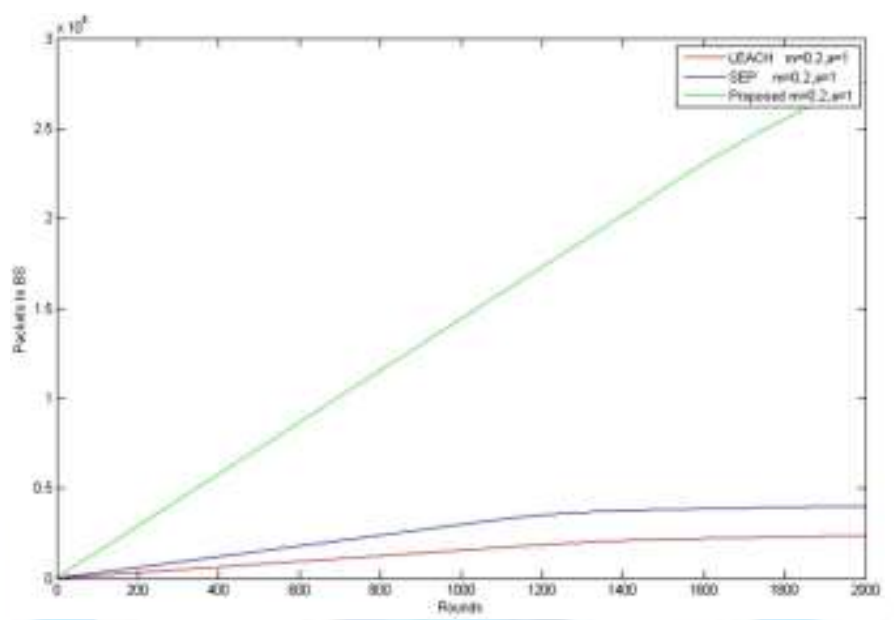

Fig. 8. Throughput of LEACH, SEP and proposed method TABLE III. COMPARISON TABLE WHEN M=0.1 AND A=2

\begin{tabular}{|l|l|l|l|}
\hline Protocol & $\begin{array}{l}\text { Stability Period } \\
\text { (Rounds) }\end{array}$ & $\begin{array}{l}\text { Network } \\
\text { Lifetime } \\
\text { (Rounds) }\end{array}$ & $\begin{array}{l}\text { Throughput } \\
\text { (Packets) }\end{array}$ \\
\hline LEACH & 899 & 5583 & $2.44 \times 104$ \\
\hline SEP & 1150 & 5078 & $4.02 \times 104$ \\
\hline proposed method & 1584 & 5966 & $2.26 \times 105$ \\
\hline
\end{tabular}

\section{CONCLUSION}

The proposed work introduced novel technique for managing sensor nodes in WSN environment. This method utilize base station information for deciding whether data will be sent to directly from node to base station or data will be sent via clustering method. Any node near to base station not need to form cluster hence energy of node utilized properly. All node far from base station send data trough cluster formation and $\mathrm{CH}$ will aggregate data of all member belong to current cluster then send combine data to base station. Network consists of advance node with more energy than normal nodes and these nodes deploy as far distance from base station to utilize their energy properly. The proposed method is able to improve $50 \%$ energy utilization by effective energy management. Overall results are effective on basis of energy utilization and throughput of network is batter with compare to LEACH and SEP methods.

\section{REFERENCES}

[1] Heinzelman, W. R., Chandrakasan, A., \& Balakrishnan, H. (2000, January). Energy-efficient communication protocol for wireless microsensor networks. In System Sciences, 2000. Proceedings of the 33rd Annual Hawaii International Conference on (pp. 10-pp). IEEE.

[2] Smaragdakis, G., Matta, I., \& Bestavros, A. (). SEP: A stable election protocol for clustered heterogeneous wireless sensor networks. Boston University Computer Science Department, 2004.

[3] W. Heinzelman, A. Chandrakasan,H. Balakrishnan, Energy-efficient communication protocol for wireless microsensor networks, in: Proceedings of the 33rd Hawaii International Conference on System Sciences (HICSS'00), January 2000.

[4] S. Lindsey, C.S. Raghavendra, PEGASIS: power efficient gathering in sensor information systems, in: Proceedings of the IEEE Aerospace Conference, Big Sky, Montana, March 2002.

[5] Manjeshwar, D.P. Agrawal, TEEN: a protocol for enhanced efficiency in wireless sensor networks, in: Proceedings of the 1st International Workshop on Parallel and Distributed Computing Issues in Wireless Networks and Mobile Computing, San Francisco, CA, April 2001.

[6] Manjeshwar, D.P. Agarwal, APTEEN: a hybrid protocol for efficient routing and comprehensive information retrieval in wireless sensor networks, in: Parallel and Distributed Processing Symposium. Proceedings International, IPDPS 2002, pp. 195-202.

[7] Ying Liao, Huan Qi, and Weiqun Li, "Load-Balanced Clustering Algorithm with Distributed Self-Organization for Wireless Sensor Networks", IEEE Sensors Journal, Vol. 13, No. 5, May 2013. 


\section{ISSN 2277-3061}

[8] M. Chatterjee, S. K. Das, and D. Turgut, "WCA: A weighted clustering algorithms for mobile ad hoc networks,"Cluster Comput., vol. 5, no. 2, pp. 193-204, 2002.

[9] Y. Fernandess and D. Malkhi, "K-clustering in wireless ad-hoc networks," in Proc. 2nd ACM Workshop Principles Mobile Comput. Conf., Oct. 2002, pp. 31-37.

[10] M. Lehsaini, H. Guyennet, and M. Feham, "A novel cluster-based selforganization algorithm for wireless sensor networks," inProc. Int. Symp. Collabor. Technol. Syst. Conf., May 2008, pp. 19-26.

[11] Ying Liao, Huan Qi, and Weiqun Li, "Load-Balanced Clustering Algorithm With Distributed Self-Organization for Wireless Sensor Networks", IEEE SENSORS JOURNAL, VOL. 13, NO. 5, MAY 2013

[12] Anuja Modi, "WSN Performance Issues and Various Clustering Methods", International Journal of Computer Applications, Volume - No. -, pages IJCA, 2014. 